\title{
Quantification of fracture properties and microstructural features of roasted Marcona almonds by image analysis
}

\section{Paula Varela, José Miguel Aguilera and Susana Fiszman}

anstituto de Agroquímica y Tecnología de Alimentos (CSIC). Apartado de Correos 7346100 Burjassot, Valencia, Spain

${ }^{\mathrm{b}}$ Departamento de Ingeniería Química y Bioprocesos. Pontificia Universidad Católica de Chile. P.O. Box 306. Santiago 22, Chile

Received 27 November 2006; revised 1 February 2007; accepted 9 February 2007. Available online 5 March 2007.

\begin{abstract}
A method to quantify fracture pattern and microstructural heat damage through image analysis was proposed. The microstructure of Marcona almonds with different degrees of roasting $\left(200^{\circ} \mathrm{C} ; 0,1.5,3,4.5,6 \mathrm{~min}\right)$ was related with their compression and fracture behaviour. Mechanical tests were performed with a TAXT2 texture analyser. The fracture pattern was characterized by measuring the particle size distribution with image analysis after compression. Light microscopy (LM) and scanning electron microscopy (SEM) were used to observe the changes in the microstructure of the almonds during roasting. The use of image analysis to quantify micro- and macrostructural features proved to be a very useful tool for analysing the crispness/crunchiness behaviour of the samples. The disruption of the inner parenchyma in the samples roasted for longer times proved to be the principal cause of their increased brittleness. The heterogeneity caused by heat degradation contributed to the failure of the material under compression, changing it from a deformable hard solid (raw sample) to a brittle one with enhanced crispy/crunchy characteristics.
\end{abstract}

Keywords: Almonds; Image analysis; Microstructure; Fracture analysis; Texture 


\section{Introduction}

Almonds are one of the most popular tree nuts worldwide and Spain is the world's second-biggest almond producer (Dourado, Barros, Mota, Coimbra, \& Gama, 2004; FAO, 2005). Their cultivation has increased in recent years, particularly in the southeastern region where the principal variety is Marcona, which is mostly consumed as a roasted or fried snack. Roasted almonds have a crisp/crunchy texture. Convection, the most common roasting system, causes changes in the appearance, flavor and, particularly, textural properties (crispness, hardness, grittiness, porosity, fracturability, etc.) of almonds, due to dehydration, browning, lipid oxidation and diverse structural changes (Gou, Guerrero, Valero, Arnau, \& Romero, 2000; Pascual-Albero, PérezMunuera, \& Lluch, 1998; Varela, Chen, Fiszman, \& Povey, 2007).

Since Szczesniak and Klein (1963) highlighted the importance of food crispness for consumers, different approaches have been used to evaluate it: sensory tests, various instrumental texture methods and sound analysis, as well as microstructural observation. Crispness and crunchiness are directly related to the mechanical and fracture properties of solid food materials, to their macro- and microstructure and to the way they are eaten (Vincent, 1998). Microstructure has a considerable impact on crispness, brittle fracture and sound generation and propagation (Aguilera, 2006). Different microstructural features (structural irregularities and existing cracks, crack stoppers, water or oil distribution and anisotropy of the structure) perform different roles in fracture and crack propagation through the material. However, the contribution of the specific structural components of the food product to its texture and their relative importance in the perception of crispness is still unknown, as few scientists have focused on the relation between the physical, sensory and structural properties of crispy products (Duizer, 2001; Lillford, van Vliet, \& van de Velde, 2006). Pascual-Albero et al. (1998) performed a qualitative study of the microstructure of Marcona almonds after roasting, using scanning electron microscopy (SEM), and observed a loss of cellular organisation and an alteration of the cytoplasmic network surrounding the lipid and protein bodies, resulting in decompartimentalization of the cell material. Saklar, Ungan, and Kantnas (2003) performed similar qualitative observations by light microscopy in roasted hazelnuts. Varela et al. (2007) proposed that the sensory crispness, fracture mechanics and sound emission of Marcona almonds change substantially with roasting time and 
are related to their microstructure, as observed by environmental SEM. Image analysis (IA) of digital photomicrographs makes it possible to obtain quantitative information through computer technology. IA allows microstructural changes to be evaluated at different levels of resolution, leading to an objectivation of microscopy through the conversion of images to numerical data (Aguilera \& Stanley, 1999; Kaláb, AllanWojtas, \& Miller, 1995). Image analysis involves image acquisition, pre-processing operations, image segmentation, feature extraction and interpretation of the extracted features - counting particles, calculating geometric properties such as area, length, perimeter, etc. measuring colour, etc. (Aguilera \& Briones, 2005; Du \& Sun, 2004; Pedreschi, Mery, Mendoza, \& Aguilera, 2004).

The aim of this work was to study the microstructure of Marcona almonds with different degrees of roasting, by means of quantitative image analysis, and the relationship between their microstructure and fracture mechanics, studied by macroimage analysis.

\section{Materials and methods}

\subsection{Testing materials}

Raw, peeled marcona almonds (Prunus amygdalus L.), purchased in a local supermarket in Valencia (Spain), were divided into five lots. One was left raw and four were roasted in a convection oven at $200{ }^{\circ} \mathrm{C}$ for $1.5,3,4.5$ and 6 min. After cooling, all the samples were stored in airtight containers (rigid polypropylene) and placed in a refrigerated chamber until they were analysed. The almonds were selected by size and shape to ensure minimum variation within a batch. Their dimensions were: length $22 \pm 1 \mathrm{~mm}$, width $14 \pm 1 \mathrm{~mm}$, height $7 \pm 1 \mathrm{~mm}$ and weight $1.2 \pm 0.1 \mathrm{~g}$.

\subsection{Fracture analysis}

\subsubsection{Compression test}

A TAXT2 Texture Analyser (Stable Micro Systems, Godalming, UK) with a 40-mm diameter cylinder probe $(\mathrm{P} / 40)$ was used for the compression tests. The test settings were: test speed $1 \mathrm{~mm} / \mathrm{s}$, target mode: distance, distance $5 \mathrm{~mm}$, trigger $10 \mathrm{~g}$. Six 
replications were performed (six almonds from each of the five lots). The compression distance was the maximum possible to fracture the samples into pieces but to avoid overloading the texture analyser.

\subsubsection{Fracture pattern quantification by image analysis}

After fracturing the almonds in the texture analyser, the pieces were carefully transferred to an open Petri dish to be photographed. A colour digital camera, model CoolSnap-Pro ${ }_{\mathrm{cf}}$ (Media Cybernetics, USA), connected to a computer interface, was mounted on a stand $9 \mathrm{~cm}$ from the sample, which was placed on a black surface. Uniform fluorescent lighting was used to illuminate the samples. The angle between the camera lens and the sample was $90^{\circ}$. No zoom and no flash were used.

Image Pro-Plus 4.5 imaging software (Media Cybernetics, USA) was used to analyse the photographs. They were converted to grey scale, pre-processed and segmented using histogram-based segmentation. When slightly connected objects were observed in the image, they were manually resolved so that they could be counted as individual objects. In the binary images, objects were counted and areas were measured.

\subsection{Microscopic evaluation of changes during roasting}

\subsubsection{Light microscopy (LM)}

Tissue sections were obtained from the centre of the almond (transversal cuts). Two almonds from each batch were studied. The sections were fixed for $48 \mathrm{~h}$ by immersion in a fixative solution ( $5 \%$ formaldehyde, $5 \%$ acetic acid, $90 \%$ ethanol $70 \%$ ).

Dehydration of the tissue sections was accomplished by immersion in a graded series of aqueous ethanol $(50 \%, 70 \%, 95 \%$ and $100 \%)$ for $30 \mathrm{~min}$ each, ethanol 100\% for $15 \mathrm{~min}$ and tert-butanol for $8 \mathrm{~h}$. The dehydrated tissue was then embedded in wax (Paraplast Plus, melting point $58^{\circ} \mathrm{C}$ ). After hardening, blocks were obtained and thin sections $(20 \mu \mathrm{M})$ for LM were cut with a microtome (Jung, Germany). Ribbons of sections were collected on a glass slide, which has been cleaned and treated with an albumin/glycerin solution to aid adhesion. Slides were placed on a warming tray $\left(40{ }^{\circ} \mathrm{C}, 3 \mathrm{~h}\right)$ to allow the sections to flatten, the water to evaporate and the sections to adhere to the slide. The tissue sections were deparaffinized in xylene ( 3 baths, 10 min each bath), hydrated to 
water using an ethanol series $(100 \%, 95 \%, 70 \%$ and 50\%, 5 min each step, ending with water for $1 \mathrm{~min}$ ). The washed sections were stained for $30 \mathrm{~min}$ with a safranin solution ( $0.5 \mathrm{~g}$ safranin, $50 \mathrm{ml}$ ethanol $95 \%$ and $50 \mathrm{ml}$ distilled water). They were washed in water until no more stain could be removed from the sections. This was followed by dehydration in ethanol $(50 \%, 70 \%, 95 \%$ and $100 \%, 5$ min each step). Then sections were stained for 3 min with a fast green solution $(0.1 \mathrm{~g}$ fast green, $10 \mathrm{ml}$ absolute ethanol, $10 \mathrm{ml}$ eugenol). To activate the colours, the sections were washed twice (10 min each) with an eugenol solution (50 ml eugenol, $25 \mathrm{ml}$ absolute ethanol, $25 \mathrm{ml}$ xylene). Sections were finally cleared in three changes of $100 \%$ xylene (10 min each) and air dried. A coverslip was mounted with Permount to make a permanent preparation.

Safranin stains lignified, thick walled structures (i.e. vascular tissue) red; while fast green stains cytoplasm and cellulose-containing cell walls (i.e. parenchyma cells) green. Both types of structures might be responsible for textural changes in almonds due to roasting. The stained tissue was observed under an Olympus BX50 light microscope (Olympus Corporation, Japan) with $\times 4$ and $\times 20$ magnifications. A CoolSnap-Pro ${ }_{\text {cf }}$ (Media Cybernetics, USA) colour digital camera was attached to the microscope to obtain the images. Two almonds from each roasting time were studied; from each of these samples six sections were obtained. A total of 120 photographs were taken.

\subsubsection{LM image analysis}

Using Image Pro-Plus 4.5 imaging software, colour light microscopy photographs, magnified $\times 4$, were converted to 8-bit grey scale images then pre-processed and segmented using histogram-based segmentation. The histogram of the binary image was obtained and the percentage of white was calculated. In order to estimate the extent of parenchymatic disruption - empty cells and widened intercellular spaces - the percentage of white areas in the image of the raw sample (no disruption) was subtracted from the percentage of white areas measured in the roasted samples.

\subsubsection{Scanning electron microscopy (SEM)}

SEM was performed with a LEO1420VP SEM in conventional mode (Leo Electron Microscopy Inc., Thornwood, NY). The surfaces of the raw and 6 min roasted samples 
were metallised with Au/Pd (Pelco 91000 Sputter Coater, Ted Pella, Redding, California). A $20 \mathrm{kV}$ accelerating voltage was used for the observation. Images were captured as grey scale digital files (1024×768 pixels, 8 -bit).

\subsection{Statistical analysis}

One-way analysis of variance was performed on the parameters measured by image analysis, using the Statgraphics Plus 2.1 package (Bitstream, Cambridge, MA), to assess differences due to roasting.

\section{Results and discussion}

\subsection{Compression test}

Fig. 1 shows three examples (raw, 3 and 6 min roasted samples) of the force vs. displacement curves during compression. In the first part of the curves, the force increased almost linearly with the displacement as the sample was experiencing deformation but no structural breakdown occurred. This region is characteristic of the elastic behaviour of solids and is a function of the rigidity (slope), shape and size of the tested material. Roasting caused a decrease in rigidity (lower initial slope values). A sudden decrease in the force value marked the first structural breakdown, at about $150 \mathrm{~N}$ in the raw sample and at about $50 \mathrm{~N}$ and a lower strain in the 6 min roasted almond. Depending on the brittleness of the material, this initial fracture propagated until inhibited by the presence of a crack stopper, then started again as the almond was further deformed. The 6 min roasted almond was clearly more brittle: it fractured at a lower deformation and the force curve presented many sudden drops as deformation increased, reflecting fractures of rapid propagation and stop (Varela et al., 2007). The $4.5 \mathrm{~min}$ roasted almond presented a force-distance profile similar to the $6 \mathrm{~min}$ roasted sample, while the behaviour of the 1.5 min roasted sample was intermediate between those of the raw and 3 min samples (not shown).

Fig. 1. Compression test. Force $(\mathrm{N})$ versus distance $(\mathrm{mm})$, raw (dotted line), 3-minute roasted (grey line) and 6 min roasted almonds (black line). Fig. 1. Compression test. Force $(\mathrm{N})$ versus distance $(\mathrm{mm})$, raw (dotted line), 3-minute roasted (grey line) and 6 min roasted almonds (black line). 


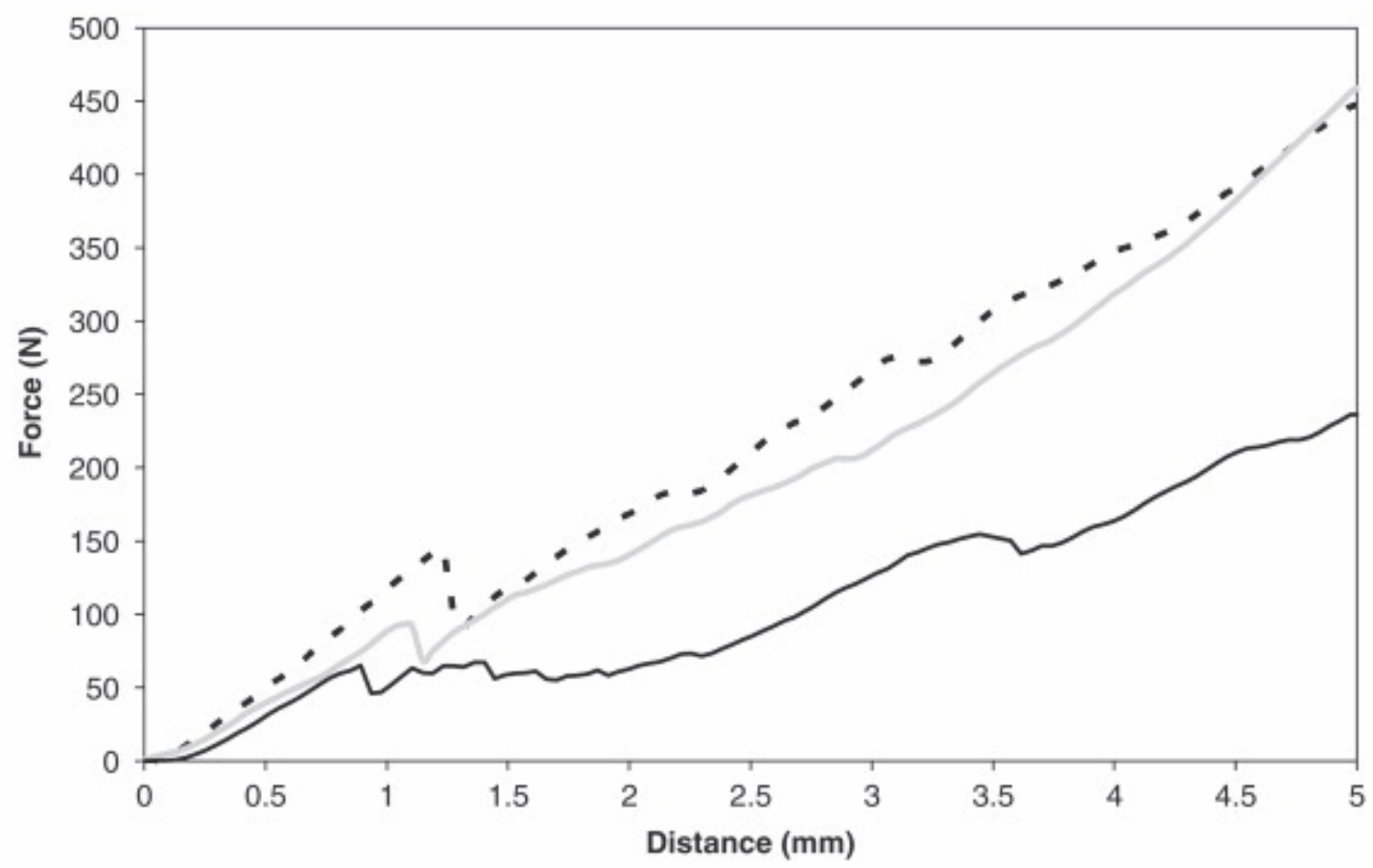

\subsection{Fracture pattern analysis}

Fracture mechanics depend not only on the parameters of the compression test, but also on the shape, size, and structure of the sample, and on the different levels of its microstructure (cellular bodies, cells, tissues). In a previous work, Varela et al. (2007) qualitatively observed large visual differences in the breakage pattern after compression of roasted Marcona almonds. In the present study, quantification of this phenomenon was accomplished by image analysis. Images of fragments produced by compression are shown in Fig. 2(a). After segmentation and binarization, the objects of interest (fragments) were clearly identified and their individual characteristics (number and size) could be measured. Area is the size feature most frequently used to characterize objects. In digital images it is directly determined by counting the number of pixels that form the object, which, after calibration, can be converted to area units (Aguilera \& Germain, 2007). 


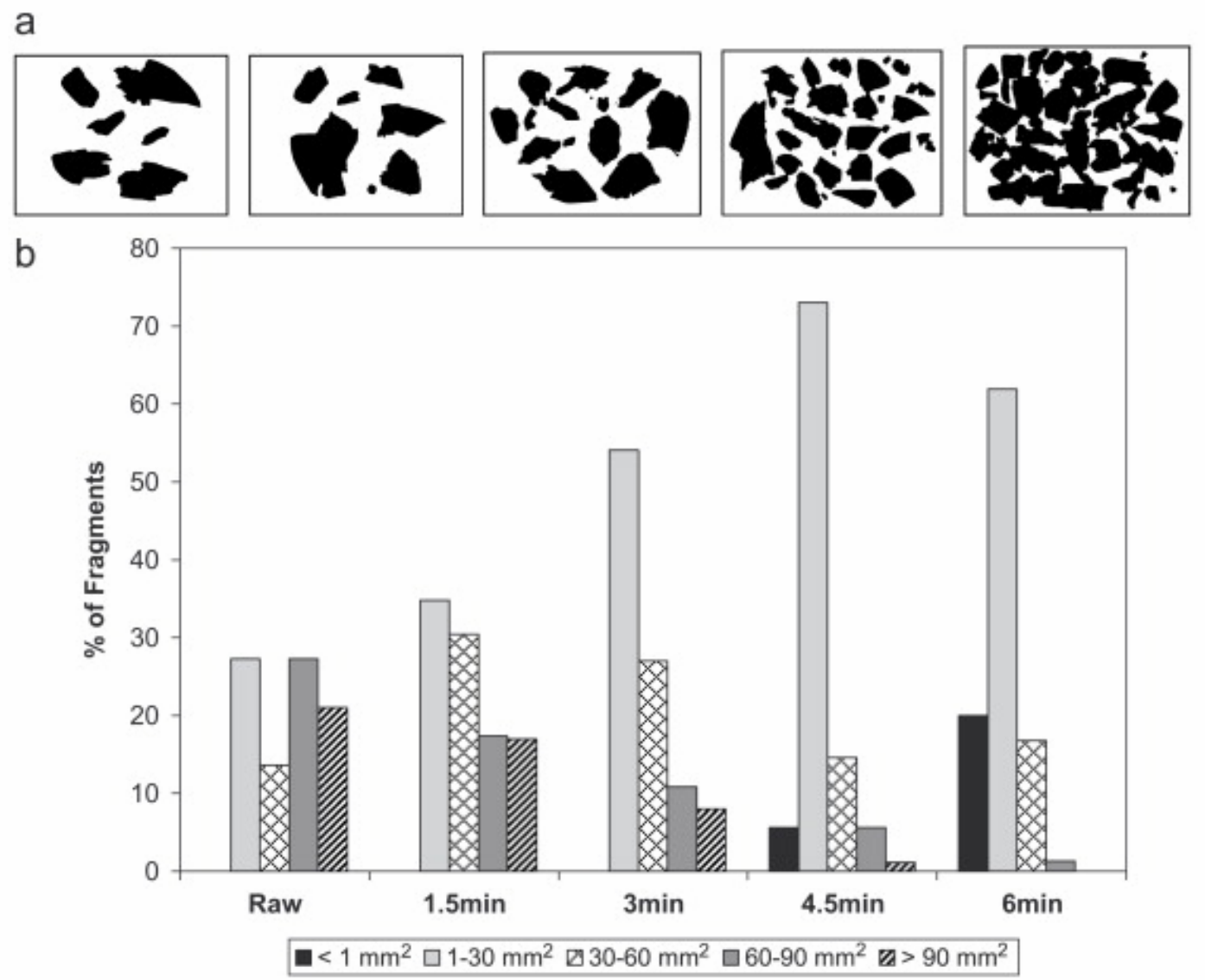

Fig. 2. (a) Binary images of fragments of samples after fracture by compression, from left to right: raw, 1.5, 3, 4.5 and 6 min roasted sample. (b) Fragment size distribution after fracture of almonds with different roasting times (calculation by image analysis).

The features extracted from the photograph of each sample compressed were the number of fragments and the average area of the fragments (Table 1). Significant differences between the samples were found in both parameters. Fig. 2(b) shows the size distribution of fragments. As roasting time increased, the fracture produced more and smaller fragments because of the increased brittleness of the samples due to the heating process.

Table 1.

Parameters extracted from fracture image analysis 


\begin{tabular}{|l|l|l|}
\hline Sample & Number of fragments & Average fragment area $\left(\mathbf{m m}^{2}\right)$ \\
\hline Raw & $6^{\mathrm{a}}$ & $57.2^{\mathrm{a}}$ \\
\hline $1.5 \mathrm{~min}$ & $6^{\mathrm{a}}$ & $58.4^{\mathrm{a}}$ \\
\hline $3.0 \mathrm{~min}$ & $9^{\mathrm{a}}$ & $45.9^{\mathrm{b}}$ \\
\hline $4.5 \mathrm{~min}$ & $22^{\mathrm{b}}$ & $20.8^{\mathrm{c}}$ \\
\hline $6.0 \mathrm{~min}$ & $39^{\mathrm{c}}$ & $13.8^{\mathrm{c}}$ \\
\hline
\end{tabular}

Identical letters indicate that there is no significant difference at $p>0.05$ (Fisher's LSD).

The procedure to quantify the fracture characteristics of a solid food sample proposed in this work is simple and is representative of the material failure. To the best of the authors' knowledge, this is the first time that image analysis has been used in a food material to quantify particle size distribution after compression.

\subsection{LM}

The compression curves obtained for the roasted samples were characteristic of a crispy/crunchy product, showing multiple fracture events that were probably the result of a non-homogeneous structure. While microstructural features such as water or oil phase distribution cause anisotropy in the food material, the particular importance of these factors in fracture behaviour and in the perception of crispness/crunchiness is still unknown (Gou et al., 2000; Lillford et al., 2006; Varela et al., 2007; Vincent, 1998).

It was hypothesised that the differences in the breakage pattern between the samples might be based in microstructural changes. To study this, light microscopy of stained transversal cuts was performed. As already described in previous works (PascualAlbero et al., 2003; Young, Schadel, Patee, \& Sanders, 2004), the peeled almond cotyledon microstructure, viewed in cross section, consists of an epidermis formed by a single layer of long cells covering the parenchyma; the epidermis is similar on the outside and on the flat, inner surface of the cotyledon. The parenchyma is composed of thin-walled isodiametric cells and the majority of its components are protein and lipid bodies. Fig. 3(a) shows a LM image of the cross section of a raw almond: the cells are compactly packed, only few intercellular spaces can be observed and the external epidermis is intact. Fig. 3(b) shows a LM photograph of a 6 min roasted almond: extensive damage to the external epidermis can be observed, the long-celled single layer 
is missing, as are some of the parenchymatous sub-layers, and some channels have formed between the cells, presumably to let the vapour formed by heating escape from the almond. Pascual-Albero et al. (2003) had already reported this kind of damage in the cells near the surface: on exiting the cells, water vapour exerts pressure, causing failures in the food material and leading to the exit of extra cytoplasmic material (coalesced lipids and proteins). The damage in the inner layers of the parenchyma has not been reported previously, however, and was even more extensive than the external damage in the roasted almonds. This phenomenon may be observed in Fig. 3(b) but can be seen more clearly in the close-up pictures, shown in Figs. 3(c) and (d), where the parenchyma of the raw and 6 min roasted samples can be compared. In the 6 min roasted sample, the consequences of the heating process were very extensive: the cell shape changed, some of the cell walls were ruptured and many of the cells were empty, with cellular material lost into the intercellular spaces; the size and shape of the protein bodies also changed, probably as a result of denaturation. The rupture of the cell walls was presumably caused by the internal pressure generated by vapour trying to exit from the cells. Saklar et al. (2003) reported a similar fact in roasted hazelnuts, which suffered damage near their internal cavity during roasting, also observing disruption of the cytoplasmatic network and decompartmentalization of cell material. 

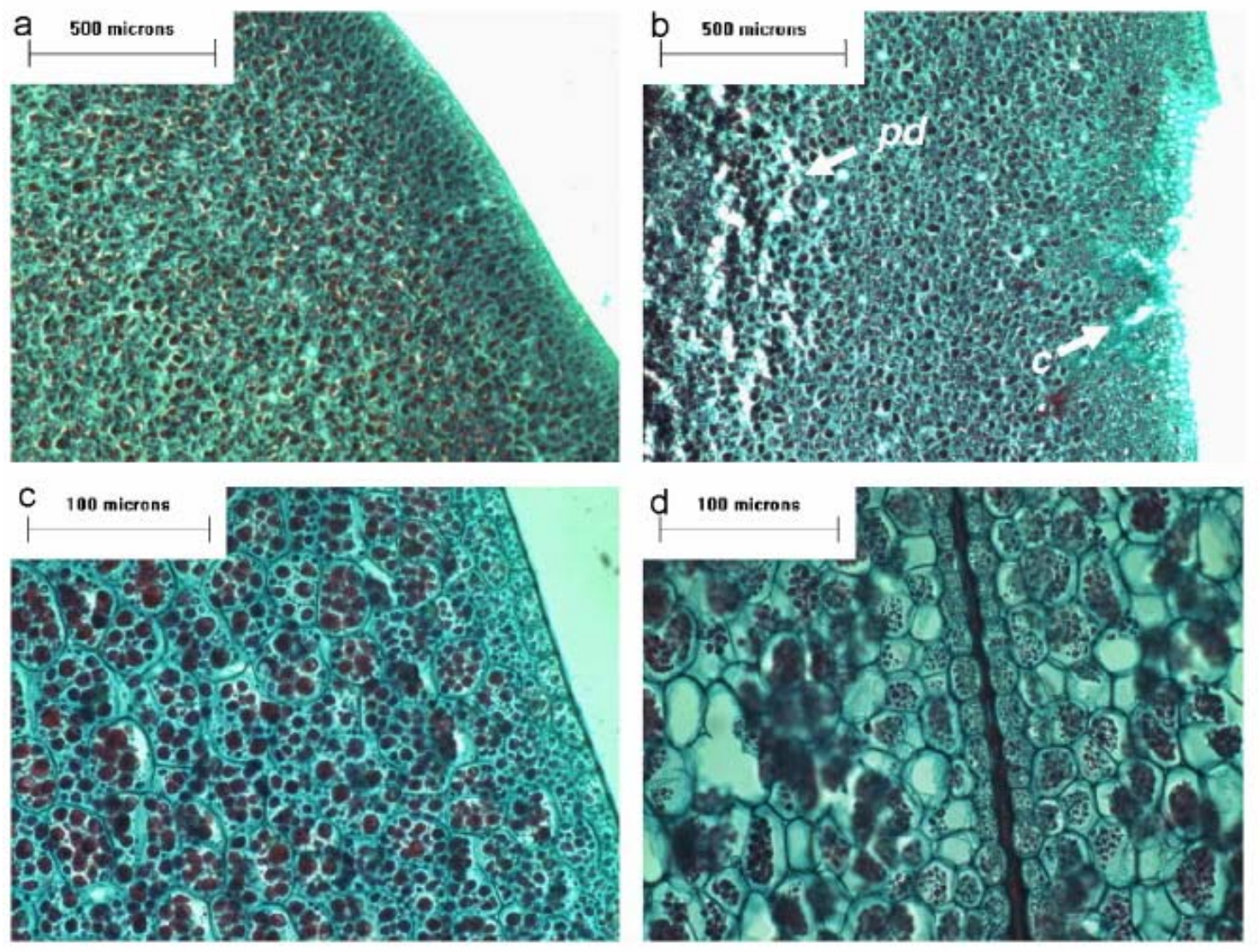

Fig. 3. Light microscopy. Outer epidermis and parenchyma $(\times 4)$ : (a) raw almond, (b) 6 min roasted almond. Inner epidermis and parenchyma (x20): (c) raw almond, (d) 6 min roasted almond. $p d$ : parenchyma disruption, $c$ : channels. Note: cotyledons in (c) became separated during the staining process while in $(d)$ they remained together.

While the damage in the external cytoplasm and epidermis could already be observed in 1.5 min roasted almonds and was of a comparable extent for all roasting times, the disruption of the inner layers of the parenchyma was qualitatively observed only in the 4.5 and 6 min roasted samples.

\subsubsection{LM image analysis}

Changes in the microstructure that led to different fracture patterns were quantified. A method to estimate the extent of the disruption of the internal parenchyma by image analysis is proposed.

LM images were taken from the parenchyma close to the inner epidermis (four photographs for each roasting time and for the raw sample). As an example of the method, Fig. 4(a) shows one of the LM pictures and Fig. 4(b) shows the same image binarised (only black and white pixels) after processing and segmentation, ready for 
image analysis. In the roasted samples, the disrupted zone appeared in white in the binary image. The raw sample also contained some regions in white, corresponding mostly to small intercellular spaces and vascular tissue. In order to estimate the extent of parenchymatic disruption - empty cells and empty intercellular spaces- the percentage of white areas in the image of the raw sample (no disruption) was subtracted from the percentage of white areas measured in the roasted samples:

$$
\begin{aligned}
& \% \text { parenchyma disruption }=\% \text { white } \\
& \quad \text { roasted sample } \\
& \quad \% \text { white }_{\text {raw sample }}
\end{aligned}
$$
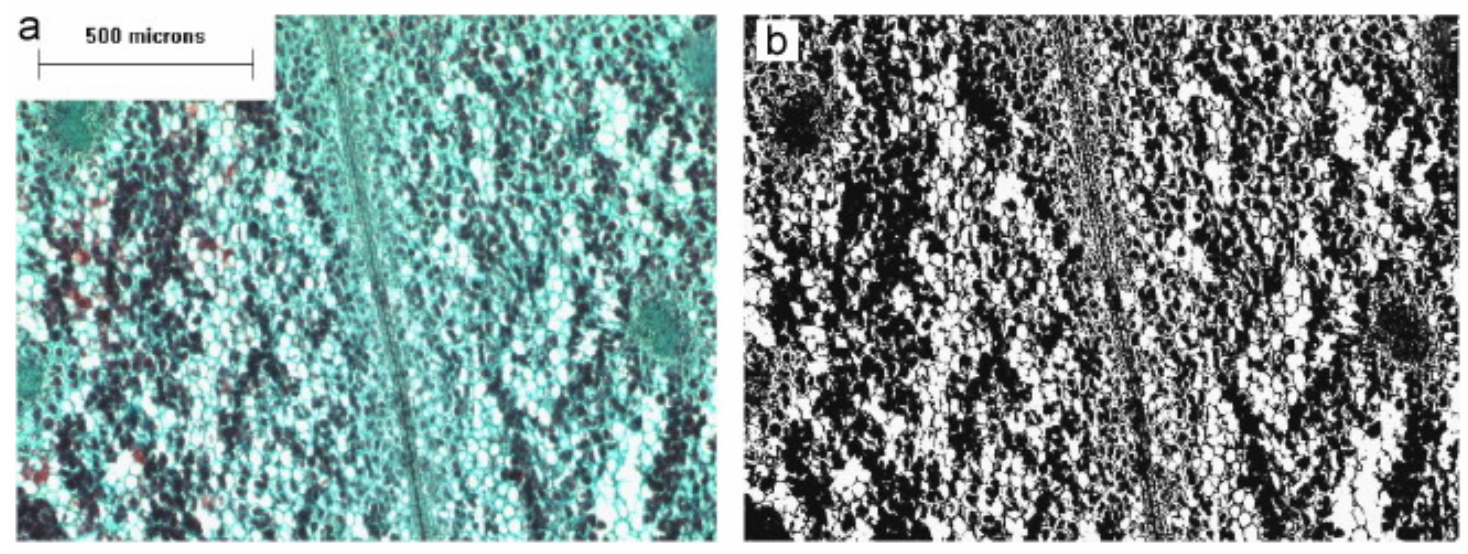

Fig. 4. (a) Light microscopy of a 6 min roasted almond, inner epidermis and parenchyma $(\times 4)$. (b) Same image as in (a) after image processing and binarization.

Fig. 5 shows the results of the calculation. In the samples roasted for $3 \mathrm{~min}$ or less, there is barely any evidence of disruption. In samples of 4.5 and 6 min roasting, as much as $30 \%$ of the parenchyma close to the inner surface of the cotyledon is disrupted. 


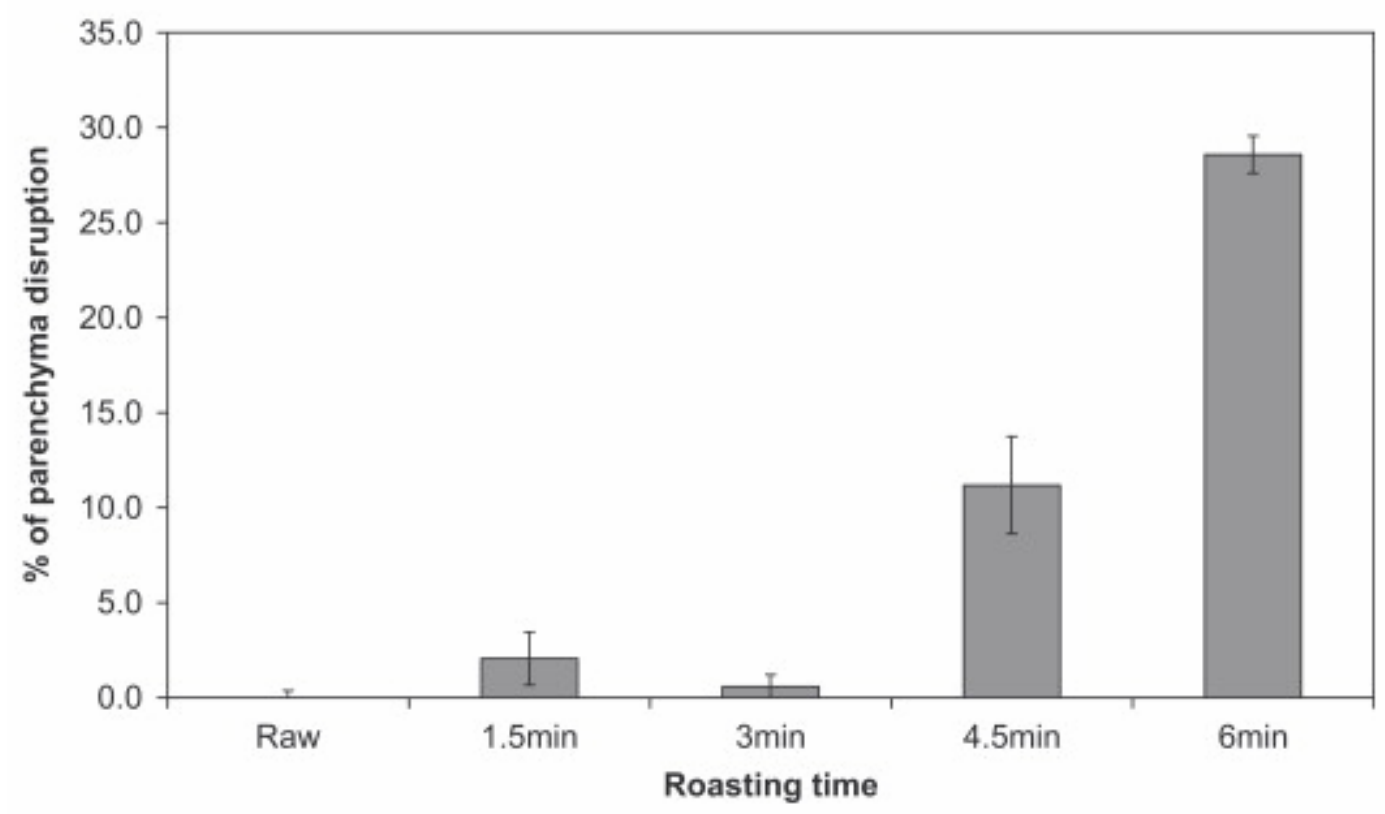

Fig. 5. Percentage of parenchyma disruption estimated by image analysis of the light microscopy image. Bars are the standard deviation.

From the results obtained in the compression, fracture and LM studies, it may be stated that parenchyma disruption is the principal cause of the rise in brittleness in the 4.5 and 6 min roasted samples. The heterogeneity caused by this disruption in the roasted samples may have produced several zones where fracture could start. The material failed under compression, changing from an elastic, resistant solid (raw sample) to a brittle one (more roasted samples) with enhanced crispness/crunchiness. These results confirm the hypothesis of the miscrostructural origin of the structural failure.

\subsection{SEM}

SEM permitted a more detailed study of the fissures in the surface of the almonds. Fig. 6(a) shows the external surface of a raw almond. The peeling process produced the rough surface; however, there are no visible fissures or cracks. Fig. 6(b) shows the external surface of a 6 min roasted almond. The surface is similar to the raw one, but a long crack is visible. In Fig. 6(c), a closer look at that same crack makes it possible to see the interior, showing a disrupted, disorganized parenchyma with groups of cells and cell material delimiting channels in the almond material formed by the vapour leaving the sample during the roasting process. Fig. 6(d) shows another fracture in the surface 
of the 6 min roasted sample: the fissure is incipient and it may be seen how the formation of the crack is accompanied by the exit of cell material.
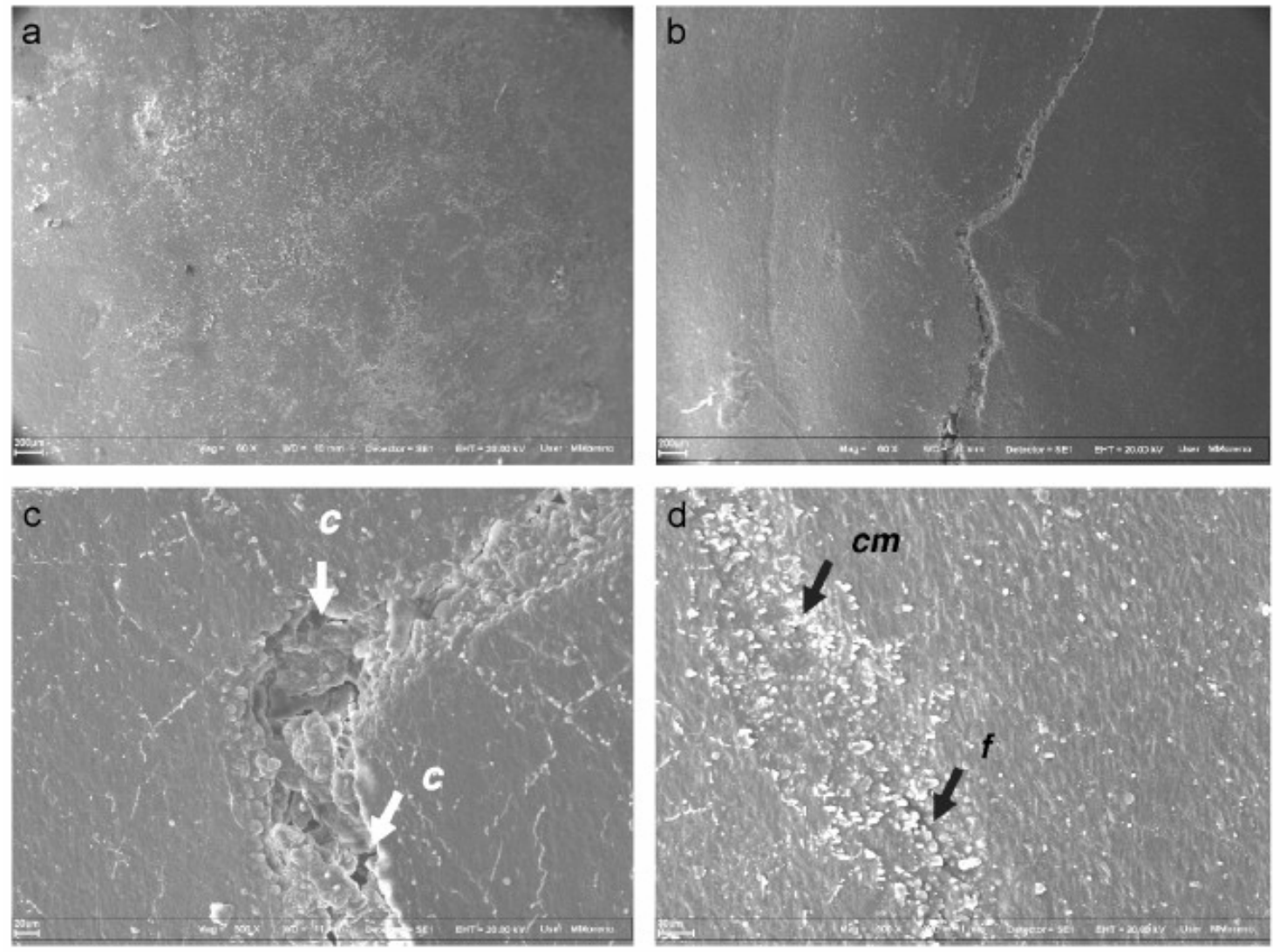

Fig. 6. Scanning electron microscopy. External surface (a) raw almond; (b)-(d) 6 min roasted almond. $c$ : channels, $f$ : fissure, $\mathrm{cm}$ : cell material.

\section{Conclusions}

The proposed image analysis methods were successfully used to quantify the features of both macro and microphotographs related to the fracture of roasted almonds.

The particle size distribution of the fractured samples, calculated by image analysis, would appear to be a very useful tool for characterizing the fracture features of crispy/crunchy foods such as other nuts, snacks, biscuits, etc.

LM and SEM photographs revealed the thermal damage in the samples during the roasting process; this damage was also quantified efficiently by image analysis. The application of this method could be extended to other food samples with tissue damage caused by such processes as heat, high pressure, microwaves, electrical pulses, etc. 
Parenchyma disruption proved to be the principal cause of enhanced brittleness in the samples roasted for longer times. The heterogeneity caused by this disruption made the material fail under compression, as it caused it to change from an elastic, resistant solid (raw sample) to a more brittle one with improved crispy/crunchy texture characteristics.

\section{Acknowledgements}

The authors are indebted to the Ministerio de Educación y Ciencia (Spain) for the grant awarded to the author Paula Varela.

Funding from the Fondecyt proyect 1030913 is appreciated (Chile).

The authors would also like to thank J.C. Germain for technical assistance with image analysis.

\section{References}

Aguilera (2006) J.M. Aguilera, Structure-property relationships in low moisture products. In: P. Buera, P. Lillford, H. Corti and J. Welti-Chanes, Editors, Water properties of food, pharmaceutical and biological materials, CRC Press, Boca Raton, FL (2006).

Aguilera \& Briones (2005) J.M. Aguilera and V. Briones, Computer vision and food quality, Food Australia 57 (2005), pp. 79-87.

Aguilera \& Germain (2007) Aguilera, J.M., \& Germain, J.C. (2007). Advances in image analysis. In: D.J. McClements (Ed.), Understanding and controlling the microstructure of complex foods. (Chapter 10) UK: Woodhead Publishing Limited, in press.

Aguilera \& Stanley (1999) J.M. Aguilera and D.W. Stanley, Microstructural principles of food processing and engineering (2nd ed), Aspen Publishers Inc, Gaithersburg, MD (1999). 
Dourado, Barros, Mota, Coimbra, \& Gama (2004) F. Dourado, A. Barros, M. Mota, M. Coimbra and F. Gama, Anatomy and cell wall polysaccharides of almond (Prunus dulcis D.A. Webb) seeds, Journal of Agricultural and Food Chemistry 52 (2004), pp. 1364-1370.

Du \& Sun (2004) C.J. Du and D.W. Sun, Recent developments in the applications of image processing techniques for food quality evaluation, Trends in Food Science and Technology 15 (2004), pp. 230-249.

Duizer (2001) L. Duizer, A review of acoustic research for studying the sensory perception of crisp, crunchy and crackly textures, Trends in Food Science and Technology 12 (2001), pp. 17-24.

FAO (2005) FAO. (2005). Faostat Statistics Database. Last updated February 2005. URL:

http://faostat.fao.org/faostat/servlet/XteServlet3?Areas=\%3E862\&Items=221\&Element $\mathrm{s}=51 \&$ Years $=2005 \&$ Format $=$ Table $\&$ Xaxis $=$ Years $\&$ Yaxis $=$ Countries $\&$ Aggregate $=\&$ Cal culate $=\&$ Domain $=$ SUA\&ItemTypes $=$ Production. Crops.Primary\&language=EN. 2005 .

Gou, Guerrero, Valero, Arnau, \& Romero (2000) P. Gou, L. Guerrero, A. Valero, J. Arnau and A. Romero, Physico-chemical and sensory property changes in almonds of Desmayo Largueta variety during toasting, Food Science and Technology International 6 (2000), pp. 1-7.

Kaláb, Allan-Wojtas, \& Miller (1995) M. Kaláb, P. Allan-Wojtas and SS. Miller, Microscopy and other imaging techniques in food structure analysis, Trends in Food Science and Technology 6 (1995), pp. 177-186.

Lillford, van Vliet, \& van de Velde (2006) P. Lillford, T. van Vliet and F. van de Velde, Discussion session on solid foods, Food Hydrocolloids 20 (2006), pp. 432-437.

Pascual-Albero, Pérez-Munuera, \& Lluch (1998) M. Pascual-Albero, I. PérezMunuera and M.A. Lluch, Cotyledon structure of raw, soaked and roasted almond (Prunus amygdalus L.), Food Science and Technology International 4 (1998), pp. 189197. 
Pedreschi, Mery, Mendoza, \& Aguilera (2004) F. Pedreschi, D. Mery, F. Mendoza and J.M. Aguilera, Classification of potato chips using pattern recognition, Journal of Food Science 69 (2004), pp. E264-E270.

Saklar, Ungan, \& Kantnas (2003) S. Saklar, S. Ungan and S. Kantnas, Microstructural changes in hazelnuts during roasting, Food Research International 36 (2003), pp. 1923.

Szczesniak \& Klein (1963) A. Szczesniak and D. Klein, Consumer awareness of texture and other food attributes, Food Technology 63 (1963), pp. 74-77.

Varela, Chen, Fiszman, \& Povey (2007) Varela, P., Chen, J., Fiszman, S., \& Povey, M. (2007). Crispness assessment of roasted almonds by an integrated approach to texture description: Texture, acoustics, sensory and structure. Journal of Chemometrics in press, doi:10.1002/cem.1029.

Vincent (1998) J.F.V. Vincent, The quantification of crispness, Journal of the Science of Food and Agriculture 78 (1998), pp. 162-168.

Young, Schadel, Patee, \& Sanders (2004) C.T. Young, W.E. Schadel, H.E. Patee and T.H. Sanders, The microstructure of almond (Prunus dulcis (Mill.) D.A. Webb cv. 'Nonpareil') cotyledon, LWT_Food Science and Technology 37 (2004), pp. 317-322. 